\title{
Production Follow-Up of the LHC Main Dipoles Through Magnetic Measurements at Room Temperature
}

\author{
E. Wildner, S. Pauletta, V. Remondino, W. Scandale, E. Todesco, and C. Vollinger
}

\begin{abstract}
In this paper we review the tools used for controlling the production of the LHC main dipoles through warm magnetic measurements. For the collared coil measurements, control limits are based on the statistics relative to the pre-series production. For the cold mass, the difference between collared coil and cold mass is considered, allowing a very stringent test. In both cases, measurements are split in straight part average, variations and coil ends contributions. Two different alarm levels exist in case the measured field is out of limits. The analysis can be carried out at the manufacturer and allows detection of anomalies in the measured magnetic field. These can be either due to wrong measurements or caused by assembly defects. Techniques used to work out information on the magnet assembly from the field harmonics are outlined. We summarize the experience gathered on about 180 collared coils and 120 cold masses, pointing out the bad cases and investigating the reliability of the measurements.
\end{abstract}

Index Terms-Magnetic field errors, magnet production control, warm magnetic measurements.

\section{INTRODUCTION}

$\mathbf{T}$ HE magnetic field of all main dipoles [1] of the Large Hadron Collider will be measured at room temperature at two different stages of the production: after the collaring and after the welding of the cold mass shell. These measurements are holding points in the production, i.e., CERN must allow the continuation of the assembly or indicate corrective actions on the basis of the measurement analysis.

The holding point strategy is based on the principle of separating the control limits from the required beam dynamics targets. Control limits are worked out from a representative set of data to define the typical measured field quality with the tooling and processes that are used for the dipole production. They are defined for each measuring position. Field anomalies are singled out automatically by comparing the measured values to the control limits. Such anomalies can be due to two different reasons that cannot be separated by the automatic control: wrong measurements or assembly component errors. This analysis is to be treated case-by-case. For the cold masses we analyze the difference between the measurements of the cold mass and of the collared coil: in this way we are able to single out anomalies in the assembly procedure from collared coil to cold mass.

The quality control would have been less precise if solely based on beam dynamics targets [2], that are defined only for

Manuscript received October 20, 2003.

The authors are with the Accelerator Technology Division, CERN, Geneva Switzerland (e-mail: elena.wildner@cern.ch).

Digital Object Identifier 10.1109/TASC.2004.829037 integrated values. Indeed, the experience acquired in the production shows that assembly errors are usually limited to a few measuring positions (i.e., a few meters) along the $15 \mathrm{~m}$ magnet and therefore they barely affect the integrated values. Thus the comparison to beam dynamic targets and the corrective actions are carried out separately, as outlined in [3].

In Section II we summarize the quality control strategy. Results relative to 180 collared coils and 120 cold masses are given in Section III, and conclusions are drawn in Section IV.

\section{QUALITY CONTROL STRATEGY}

\section{A. Parameters to be Controlled}

The magnetic field components in the two apertures of a dipole are expressed as multipoles, the Fourier terms of the series expansion of the radial field component. The multipoles are given in units of $10^{-4}$ relative to the main field component and scaled to a reference radius of $17 \mathrm{~mm}$.

For both the collared coil (superconducting coils clamped in the stainless steel collars) and the cold mass (collared coil and iron yoke in the welded cylinder) the magnetic field in the aperture is measured along the magnet axis using a $750 \mathrm{~mm}$ long rotating coil [8]. Twenty consecutive measurement points are taken at room temperature with a typical excitation current of 8 to $12 \mathrm{~A}$ (cycles of negative and positive currents are alternated and results are arranged afterwards). The first and the last measurement positions are in the magnet ends and are treated separately. The positions in the "straight" part of the magnet, i.e., 2 to 19 , are considered as a homogeneous set; we have a control on the average from positions 2 to 19 and one on the variations in each position with respect to the average.

The magnetic center, i.e. the center of the coil cross section, is computed for each measurement position by the feed down of the component $b_{11}$, giving $b_{10}=a_{10}=0$. Therefore no control on $\mathrm{b}_{10}$ and $\mathrm{a}_{10}$ can be performed.

For the main field, only positions 3 to 18 are considered a homogeneous set, since also points 2 and 19 feel the head influence. Positions 1 and 20 are treated separately as usual. The field direction is analyzed separately for the ends, points 1 and 20 , and for the straight part of the magnet. No control on the field direction average is carried out since the measurement is not given with respect to the magnet reference system, which is the plane going though the center of the two apertures (best fit). Moreover, the collared coil has no rigidity: thus the measurement of the field angle is only an indication of the straightness of the support. On the other hand, angle variations along the axis 
seen on the cold mass are physically significant, since they reflect a bad alignment of the cold mass in the cylinder.

For the cold mass the analysis is done considering the difference between the cold mass and the collared coil measurements. In this way we single out anomalies in the process of the iron yoke assembly only, the collared coil anomalies having already being pointed out by the corresponding measurement. However, some features have to be pointed out. The field angle is analyzed on the cold mass and not on the difference, following the arguments of the previous section. The effect of the iron yoke is to increase the main field by a factor $k=1.18$ in the straight part and by $k=1.12$ in the coil heads. Therefore the multipole values in the cold mass are reduced by $1 / k$ and the difference is calculated according to the following expression:

$$
b_{n, c m-c c}=b_{n, c m}-\frac{b_{n, c c}}{k},
$$

where $\mathrm{cm}$ stands for cold mass and $c c$ for collared coil.

Multipolar variations along the magnetic axis can be summarized in a scalar value in microns which is called the estimated coil waviness. This is the amplitude (one sigma) of random movements of the blocks that give the closest values to the measured multipole spread according to simulations [7]. Empirical control limits are set at 30 micron and 60 microns. The coil waviness computed from the difference of cold mass and collared coil is also used to recognize if the cold mass really contains the corresponding collared coil. This check is very effective since the coil waviness allows to checking the pattern of field variations along the axis that are a fingerprint of the magnet. Finally, the coil waviness computed from the difference of two collared coil measurements is used to make sure that the new file we analyze is not by mistake a file from a previously measured magnet.

\section{B. Evaluation of the Statistics and Data Rejection}

Control limits have been first computed for the first 32 collared coils and for the first 13 cold masses excluding magnets with known peculiarities. Their values have been successively checked with more statistics, confirming their reliability. In order to estimate the quantity of data expected in the tails, a normality test has been preformed. All distributions have been found compatible with Gaussian shape with a confidence level of $95 \%$. We have the possibility to use different control bounds for different firms. In general the control bounds are the same for all firms, with the exception of the averages of some multipoles (see [2], [4] for more details).

\section{Control Bounds}

Having estimated the average and the standard deviation of the typical distribution for each parameter, we set control bounds on the average value \pm 3.5 standard deviations (warning level). In the hypothesis of a Gaussian distribution, 99.95\% of the data are within 3.5 standard deviations: this means that about one measurement of a 'normal' case over 2400 will be outside the control limits. This corresponds to having a rate of one false alarm over all the dipole production. If we had fixed the limits at 3 sigma, i.e., $99.7 \%$, we would have had $0.3 \%$ measurements of 'normal' magnets outside the control limits, i.e., 8 magnets. For the variations along the axis one has 18 times more data, and therefore the control bound is at 4 standard deviations, corresponding to about one false warning in one position over all the production. The control bounds are doubled (7 sigma and 8 sigma respectively) to point out strong anomalies (denoted as "red alarms" in the next section).

\section{QUALITY CONTROL RESULTS}

By analysing the measurement data we have to distinguish problems with the measurement and problems coming from the production (as wrong assembly, wearing out of tooling, use of faulty components). The analysis is based on an inverse approach from which the current distribution can be deduced from the field by assuming likely manufacturing errors and applying the appropriate symmetry or anti symmetry configuration. Sensitivity tables for a certain number of manufacturing errors have been calculated for the analysis [4].

\section{A. Data Overview}

On 12th October 2003, we have 194 measured collared coils (66 of Firm1, 52 of Firm2 and 76 of Firm3) and 131 measured cold masses (47 of Firm1, 29 of Firm2 and 55 of Firm3). All manufactured collared coils have been measured, whereas two cold masses (2002 and 3013) have not been measured.

We have 19 double or multiple measurements (10\% of total) for the collared coils. These are due to 6 faulty measurements to be repeated, 2 double measurements carried out to check calibration of old with new measurement system, and due to 6 collared coils that have been decollared for electrical problems. Moreover, the measurement is repeated by default when a strong anomaly is observed ( 5 cases). We observed 4 cases (2\%) of strong anomalies indicating a faulty assembly procedure that needed decollaring. This will be discussed in the next sections.

For the cold mass, we have 9 double or multiple measurements (7\% of total). These are due to 4 faulty measurements to be repeated and 3 cold masses that are unwelded for assembly faults. We also had two cases of measurement repetition due to a strong anomaly.

\section{B. Non-Standard or Faulty Measurements}

The overall rate of faulty measurements is 10 over more than 300 , i.e., around $3 \%$. This proves that the measuring system is reliable and well suited to work in the environmental condition of a manufacturer with high production rate. Since the magnetic measurements are a holding point of the assembly process, a careful follow-up of the measuring system during all the production is needed.

1) Collared Coil: Six cases of faulty measurements have been detected. In one case, the magnet was measured in the wrong longitudinal direction, and therefore the data from the nonconnection side were exchanged with the data of the connection side and vice-versa. This pattern is recognized by the statistical control, since one has an asymmetry in $a_{1}$ and $b_{3}$ values between connection and nonconnection side allowing a safe validation of data. In two cases we had problems with the measurement of the main field direction. This is caused by a wrong leveling of the measuring instrument before the measurement 
starts. In three other cases anomalies in the main field or in the transversal position of the mole have been observed. Both features disappeared after a repetition of the measurements.

2) Cold Mass: Four cases of faulty measurements and one nonstandard measurement have been detected. We had one case of inversion of the calibration files for the two measuring coils, giving an anomaly of less than 0.1 units in the shift in $b_{7, c m-c c}$ and $b_{13, c m-c c}$, which would have been extremely difficult to detect without the automatic procedure.

In one case, the field direction was wrongly measured because of a bad leveling of the measurement mole for one position (40 mrad above the average value along the magnet). The error induced an anomaly in the integrated higher order skew multipoles.

One of the measurement moles was cabled with wrong polarities, and used to measure a relevant number of cold masses. This mole was giving inverted signs for all multipoles, which have been easily detected.

We also had a wrong longitudinal positioning of the mole. Usually one should have approximately the same field in position 1 and 20, i.e., the measuring positions should be centered around the longitudinal center of the magnet. A wrong positioning by a few $\mathrm{cm}$ gives anomalies in the main field measurements of positions 1 and 20. Even though the measurement is valid, efforts are being done to correct this feature in order to preserve the homogeneity of the data set.

The only case of nonstandard measurement refers to the cold masses measured with the by-pass diode already mounted. According to the standard procedure, measurements are carried out before the diode is mounted, at positive and negative current to cancel the residual magnetization and systematic effects. In one magnet the diode was assembled before the measurements and this gave strong anomalies in the integrated skew multipoles (more than 7 sigma for $a_{2}$ and between 3.5 and 7 sigma for $a_{3}$ and $a_{4}$ ). The rest of the values were within statistics.

Some months later, a fault in the measurement system made it impossible to measure four magnets in the industry before the mounting of the diode. These magnets were measured at CERN at different currents and extrapolation of multipoles to zero field. The extrapolated values were found to be within control bounds, proving the soundness of the procedure also in this case.

\section{Non-Standard or Faulty Assembly Procedures}

1) Collared Coil: The first case of a wrong assembly procedure has been found in June 2002 at the very beginning of the pre-series (the 5th produced collared coil). At the time we had no automatic system to carry out the control of the measurements. Indeed, this was the largest anomaly found in the production up to today: the collared coil 2002 showed a 40 units spike in the main field, in one position of one aperture (see Fig. 1).

Another strong anomaly of 24 units was observed for $b_{2}$ in the same position. The case was indeed not so straightforward since inverse field calculations to trace the possible cause were not clearly indicating the origin of the problem. Moreover, the integral of the main field and the multipoles were barely affected. A decollaring was asked and revealed a double coil protection

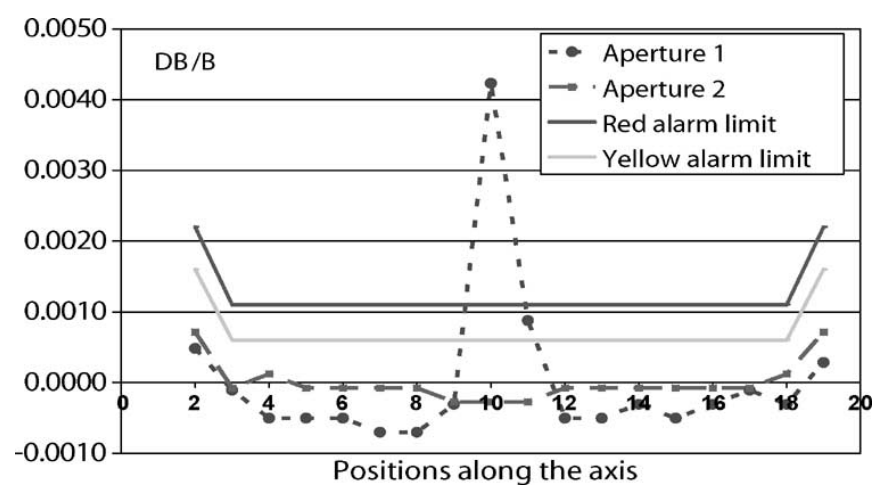

Fig. 1. Main field anomaly in collared coil 2002.

sheet $(0.5 \mathrm{~mm}$ thick). Simulations carried out a posteriori agreed rather well with the measured anomaly [4], [5].

The second case is a large variation of multipoles along the axis found in several collared coils of the early production of Firm1. The worst cases (1005 and 1006) showed anomalies up to 16 sigma in $b_{7}$, and up to 11 red alarms on several high order multipoles. The spikes were in various positions along the magnet axis. Simulations showed that this effect could be due to a random error in the positioning of all coil blocks of about $0.1-0.2 \mathrm{~mm}$. Since the amplitude of this mismatch was not large, it has been decided to proceed with the assembling. Indeed, 1006 showed no quenches in the straight part (1005 was limited by cold welds in the cable not related to this problem). A warning on the bad coil quality was anyway given to the manufacturer and the problem was traced back to a wrong procedure in the coil curing (the mould did not close before heating), and could be eliminated.

The third case was the easiest to solve, but with the smallest effect on multipoles. Collared coil 1027 showed a red alarm (10 sigma) in $b_{2}$ in one position of aperture 1 , and two yellow alarms (5 and 7 sigma) in $b_{3}$ and $a_{2}$, respectively. Simulations showed that a missing shim of $0.8 \mathrm{~mm}$ in one quadrant of the outer layer would give this effect on multipoles. Once the coils were de-collared, a missing shim was found as expected from simulations. This also allowed checking the validity of the sign conventions used in the measurements since the error was completely asymmetric. With respect to the previous case, a much larger error (four to eight times in amplitude) is giving a much lower effect on multipoles (one red alarm instead of 11, and a maximum of 10 sigma against 16). This is due to the fact that the error is located in one quadrant of the outer layer only. Therefore a larger error is needed to get the same effect on multipoles.

The last case is still open. Collared coil 2032 showed multipole anomalies in one aperture along the magnet axis of around 8 sigma in $a_{6}$ and $b_{8}$. Simulations showed that this kind of anomaly could be due to the block close to the inner layer coil pole, being shifted radially toward the cold bore of around $0.5 \mathrm{~mm}$ in one quadrant only. A measurement with a shorter measuring coil $(125 \mathrm{~mm}$ instead of $750 \mathrm{~mm})$ has been done to better estimate the size of the defect. One finds (see Fig. 2) that the spikes have a size which is much smaller then the standard length of the measuring coil, and that the size of the error is at least 50\% larger than seen from the less refined measurement. 


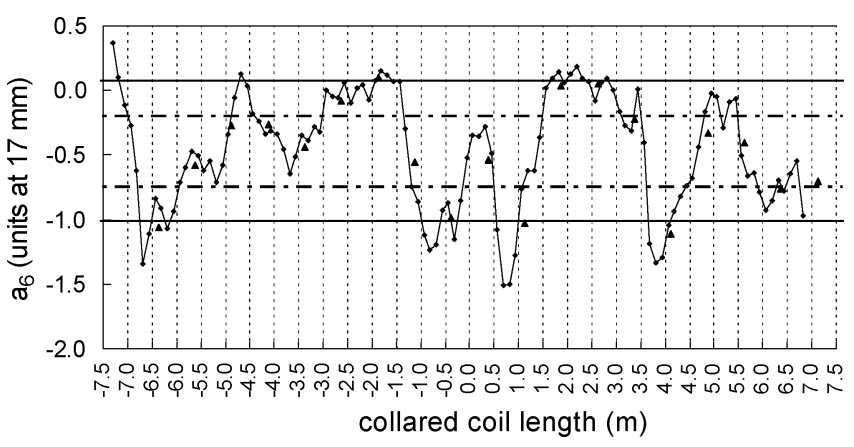

Fig. 2. Variation of $a_{6}$ along magnet axis in 2032. Measurement with $750 \mathrm{~mm}$ long rotating coil (triangles), with $125 \mathrm{~mm}$ rotating coil (dots) and control limits at 4 sigma (dashed blue lines) and at 8 sigma (solid lines).

A de-collaring has been asked. A similar anomaly has been found in 2035.

2) Cold Mass: We had two cases (1002 and 1005) of a non standard assembly procedure of the yoke, namely a different shimming of the ferromagnetic insert placed between collars and yoke. This moved the yoke insert of $0.5 \mathrm{~mm}$ outwards with respect to the center of the magnet. A change in $b_{2}$ of 6 to 8 sigma (around one unit) was detected by the statistical analysis, in agreement with simulations. The cold mass was anyway accepted since this change was not expected to affect quench performances and had a negligible impact on the overall systematic harmonics.

A few cold masses showed an anomalous variation of the field angle, due to a wrong alignment of the collared coil and the yoke laminations in the cold mass. Two cold masses were un-welded, new half-shells were used and a more careful assembly was made; a new measurement of the re-welded magnet gave normal results.

\section{CONCLUSION}

We presented an automatic procedure to screen out anomalies in the magnetic measurements at room temperature of the LHC main dipole collared coils and cold masses. The core of the method is the definition of control bounds for multipoles in each measuring position by using a sample of significant measurements. These bounds are not related to the targets for beam dynamics that are given for integrated values along the longitudinal axis and for sets of magnets.

Field anomalies can be either related to problems with faulty or non standard measurements, or caused by wrong assembly procedures. We find a low rate of faulty measurements $(3 \%)$. Four collared coils and two cold masses have shown field anomalies that have been traced back to a wrong assembly. In these cases, corrective actions have been taken, allowing curing the problem at the earliest possible stage of the assembly procedure.

\section{ACKNOWLEDGMENT}

The authors would like to acknowledge J. Billan, D. Cote, P. Galbraith and D. Giloteaux for developing the measuring system in collaboration with V. Remondino. They thank all the CERN and firm personnel involved in the magnetic measurements. A special thank to G. Peiro for the special measurement of collared coil 2032, and to L. Bottura, S. Sanfilippo, and the colleagues of the group in charge of measurements at $1.9 \mathrm{~K}$ for discussion and reciprocal help in data validation.

\section{REFERENCES}

[1] L. Rossi, , this conference.

[2] S. Fartoukh and O. Bruning, , LHC Project Report 501, 2001.

[3] E. Todesco et al., , this conference.

[4] S. Pauletta, Master thesis, Politechnico di Torino, Italy, 2002.

[5] R. Gupta, M. Anerella, J. Cozzolino, D. Fischer, A. Ghosh, A. Jain, W. Sampson, J. Schmalzele, P. Thompson, P. Wanderer, and E. Willen, "Field quality analysis as a tool to monitor magnet production," in Proceedings of MT-15, 1997, pp. 110-113.

[6] A. B. Oliva, P. Gagliardi, R. Penco, and P. Valente, "A statistical analysis of the whole ansaldo "HERA dipoles" production," Cryogenics, vol. 30, pp. 589-593, September Supplement 1997.

[7] P. Ferracin, W. Scandale, E. Todesco, and R. Wolf, "Modeling of random geometric errors in superconducting magnets with applications to the CERN large Hadron collider," Physical Review ST Accel. Beams, vol. 3, p. 122403 (1-9), 2000.

[8] J. Billan, J. Galbraith, A. Musso, and V. Remondino, , LHC Project Report 283, 2002. 